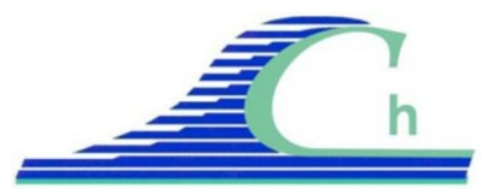

XII ${ }^{\text {ìmes }}$ Journées Nationales Génie Côtier - Génie Civil

Cherbourg, 12-14 juin 2012

DOI:10.5150/jngcgc.2012.107-A @ Editions Paralia CFL

disponible en ligne - http://www.paralia.fr - available online

\title{
Pour une caractérisation minimale des sédiments fins : application d'une démarche pour leur valorisation en matériaux routiers
}

\author{
Baptiste ANGER ${ }^{1,2}$, François THERY ${ }^{1}$, Daniel LEVACHER ${ }^{2}$
}

1.EDF R\&D, Département EPI, Site des Renardières - Ecuelles, 77818 Moret-sur-Loing Cedex, France

baptiste.anger@edf.fr ; francois.thery@edf.fr

2. Laboratoire M2C, UMR 6143 CNRS, 24 rue des Tilleuls ; Université de Caen, 14000 Caen, France

daniel.levacher@unicaen.fr

\section{Résumé :}

Face à la problématique de gestion à terre des sédiments, une démarche d'identification innovante des paramètres essentiels d'entrée dans les filières de valorisation a été entreprise. Cet article présente la démarche anticipative initiée par EDF dans le cas d'une approche matériau pour un réemploi en sous couches dans une structure routière. Cette démarche consiste à hiérarchiser par ordre d'importance les paramètres et/ou critères de caractérisation nécessaires à la mise en œuvre de la filière retenue. Une recherche des paramètres clés ou critères "déclenchant" pour cette filière est alors menée. Leur connaissance permet de tendre vers une caractérisation minimale des sédiments à valoriser.

\section{Mots-clés :}

Sédiments fins - Valorisation - Caractérisation - Technique routière - Retenues hydroélectriques - Hiérarchisation

\section{Introduction}

Depuis une décennie, la gestion des sédiments est devenue préoccupante. En effet, pour les principaux donneurs d'ordre, les pratiques usuelles de clapage en mer dans le cas des grands ports maritimes ou de mise en dépôts à terre pour les gestionnaires de voies d'eau sont de plus en plus remises en cause ou discutées. Toute immersion de sédiments dans le milieu naturel dépend de la nature et du taux de pollution et reste subordonnée à autorisation de la part de l'administration. A tout moment un refus pose le problème de leur devenir à terre. A ce titre, la réglementation évolue et place clairement la gestion des sédiments à terre dans la nomenclature ICPE (Installation Classée Pour l'Environnement), en leur conférant le statut de déchet. Confrontés à ces contraintes environnementales et réglementaires, les gestionnaires de ports, de canaux de navigation et d'ouvrages d'eau ont plus ou moins identifié des filières de valorisation voire contribué à la recherche et au développement de nouvelles voies. Bon nombre de 
travaux de recherche l'attestent mais celles-ci s'annoncent difficiles à organiser et à mettre en œuvre.

EDF en tant qu'hydroélectricien doit gérer les apports et les dépôts de sédiment dans les retenues. Il a donc initié une démarche liée à la valorisation des sédiments. Il s'agit d'une action anticipative dans le cas où les solutions de gestions actuelles (curage puis dilution, dépôt avec aménagement de terrain, remobilisation naturelle en crue, ...) ne puissent pas être appliquées.

Notons qu'en moyenne, environ $200000 \mathrm{~m}^{3}$ de sédiments sont curés chaque année sur le parc EDF (EDF/IME, 2010). Ces matériaux sont principalement restitués dans le cours d'eau et le reste est stocké à terre.

\section{Contexte}

\subsection{Origine des dépôts de sédiments de barrage}

Les sédiments proviennent essentiellement de l'érosion des bassins versants résultant du ruissellement des eaux de pluie, transportés par l'eau jusqu'aux retenues. Le ralentissement des vitesses d'écoulement de l'eau à l'approche d'un barrage entraîne la sédimentation des matériaux solides que la rivière transporte en suspension et par charriage sur le fond. L'envasement des retenues est, de ce point de vue, un phénomène inéluctable que tous les réservoirs subissent plus ou moins selon leur implantation et leur possibilité de gestion.

Pour un site donné, la nature et le volume des dépôts va dépendre d'un certain nombre de facteurs tels que la géologie des sols du bassin versant, le régime climatique de la vallée, le régime hydrologique du cours d'eau, la couverture végétale du bassin versant, son degré d'activités humaines (pratiques culturales, assainissement urbain, industries...). Il y a donc de multiples typologies de sédiments qui sont présentes dans les retenues.

\subsection{La granulométrie, une caractéristique essentielle des dépôts}

Il est souvent fait référence à une taille de $2 \mathrm{~mm}$ qui permet de qualifier le sédiment de fin $(<2 \mathrm{~mm})$ et de grossier $(>2 \mathrm{~mm})$.

Le contexte dans lequel s'exerce l'activité de production hydroélectrique reste profondément marqué par la mise en œuvre de la législation européenne, en particulier la Directive Cadre sur l'Eau (JOCE, 2000), ainsi que législation française qui en découle avec la Loi sur l'Eau et les Milieux Aquatiques (JORF, 2006). Les matériaux s'accumulant en amont de la retenue doivent être, en priorité, réintroduits dans le cours d'eau afin d'assurer le principe de continuité sédimentaire.

Dans le cadre de la valorisation à terre des sédiments, la fraction supérieure à $2 \mathrm{~mm}$ est réputée facilement valorisable. Il est vrai que les sables et graves ont longtemps été exploités dans le lit mineur des cours d'eau et dans les plans d'eau traversés. Depuis 


\section{XII ${ }^{\text {èmes }}$ Journées Nationales Génie Côtier - Génie Civil \\ Cherbourg, 12-14 juin 2012}

l'arrêté du 22 septembre 1994 relatif à l'exploitation des carrières (JORF, 1994), les extractions de granulats à des fins commerciales sont interdites.

Les sédiments fins $(<2 \mathrm{~mm})$ constituent donc la fraction à valoriser, en volumes importants et avec toutes les difficultés inhérentes aux filières actuelles envisagées et à mettre en œuvre. La démarche présentée par la suite concerne précisément ces sédiments fins, inférieurs à $2 \mathrm{~mm}$ avec un $\mathrm{d}_{50}<63 \mu \mathrm{m}$. A titre d'exemple, le tableau 1 donne la répartition granulométrique de deux sédiments prélevés à proximité de retenues hydroélectriques de bassins versants très différents. Pour ces deux retenues, une de montagne (A) et une de plaine (B), il s’agit de sédiments très fins.

Tableau 1. Répartitions granulométriques des deux retenues hydroélectriques (A-Retenue alpine ; B-Retenue de plaine)

\begin{tabular}{lllllll}
\hline Echantillons & $\begin{array}{l}>\mathbf{~ m m} \\
\text { Graviers }\end{array}$ & $\begin{array}{l}\mathbf{2 - 0 , 2} \mathbf{~ m m} \\
\text { Sables } \\
\text { grossiers }\end{array}$ & $\begin{array}{l}\mathbf{2 0 0 - 5 0} \boldsymbol{\mu m} \\
\text { Sables fins }\end{array}$ & $\begin{array}{l}\mathbf{5 0 - 2 0} \boldsymbol{\mu m} \\
\text { Limons } \\
\text { grossiers }\end{array}$ & $\begin{array}{l}\mathbf{2 0 - 2} \boldsymbol{\mu m} \\
\text { Limons fins }\end{array}$ & $\begin{array}{l}<\mathbf{\mu m} \\
\text { Argiles }\end{array}$ \\
\hline A (en \%) & 0 & 0,01 & 0,16 & 6,99 & 59,10 & 33,73 \\
B (en \%) & 0 & 1,04 & 5,85 & 14,60 & 57,00 & 21,50 \\
\hline
\end{tabular}

\subsection{Contraintes liées à l'envasement}

Les dépôts de sédiments qui s'accumulent dans les retenues sont à l'origine d'un certain nombre d'impacts. Ils affectent principalement les activités d'exploitation hydroélectriques et les usages et usagers riverains. Les effets et/ou nuisances qui résultent de ces contraintes liées à l'envasement sont listées dans le tableau 2 (EDF, 1997 et 2011).

Au regard de l'ensemble de ces contraintes, il est essentiel de porter une importance à la gestion des sédiments.

Tableau 2. Impacts liés aux dépôts de sédiments dans les retenues hydroélectriques

\begin{tabular}{ll}
\hline Catégorie d'impacts & Nuisances et effets \\
\hline \multirow{2}{*}{$\begin{array}{l}\text { Impacts sur les activités d'exploitation } \\
\text { hydroélectriques }\end{array}$} & $\begin{array}{l}\text { Obstruction de la capacité de stockage disponible de la retenue. } \\
\text { ouvrages de sécurité. } \\
\text { Limitation du marnage. }\end{array}$ \\
\hline \multirow{2}{*}{$\begin{array}{l}\text { Impact aux usages et usagers } \\
\text { riverains }\end{array}$} & Rehaussement des cotes d'eau entraînant des inondations. \\
& Problèmes visuels et/ou olfactifs. \\
& Gène pour les activités de loisir : baignade, pêche,... \\
\hline
\end{tabular}

\subsection{Le cadre réglementaire du devenir des dépôts}

Si les déblais de dragage i.e. les sédiments, sont destinés à une filière terrestre, ils entrent de fait dans la nomenclature déchets qui peuvent être à caractère dangereux ou non dangereux (article R.541-8 (annexe II) du code de l'environnement). La 
dangerosité des déchets repose sur 15 critères, notés $\mathrm{H} 1$ à $\mathrm{H} 15$ dont un seul suffit à classer le matériau comme dangereux. Des travaux sont en cours pour qualifier le caractère dangereux ou non des sédiments, notamment sur le critère H14 relatif à l'écotoxicité. La réglementation, avec le décret du 13 avril 2010 relatif aux ICPE (JORF, 2010) et sa circulaire d'application du 24 décembre 2010 (BO, 2011), évolue et place clairement la gestion des sédiments à terre dans la nomenclature ICPE. Il apparait en effet des rubriques pour les opérations de transit, de tri, de traitement, de stockage, etc.

Le cadre actuel place donc les sédiments gérés à terre comme un déchet. Mais derrière cette notion de déchets, les sédiments dragués doivent être considérés comme une ressource d'origine naturelle et renouvelable donc pérenne pour sa valorisation dans le temps, ce point de vue a été largement développé à l'étranger (PAIPAI, 1995 ; 2003 ; LEVACHER et al, 2012).

\section{Démarche d'identification des paramètres clés ou "déclenchant"}

\subsection{Constat}

Les actions de recherche ou projets déclarés sont nombreux et témoignent du besoin de création et de mise au point de filières de gestion. Ceci confirme aussi qu'il n'existe pas de voie robuste de valorisation des sédiments disponible à ce jour. Les filières les plus étudiées concernent le domaine routier, le remblaiement de carrières, de cavités et de tranchées, les aménagements paysagers, la restauration de zones humides, la valorisation agricole par amendement organique et la restructuration des sols, le renforcement de berges, la couverture définitive d'installation de stockage de déchets, et pour de moindres volumes, les matériaux élaborés par substitution d'un constituant ou liant : bétons, ciments, coulis, briques, pavés, tuiles, ... (THERY \& ANGER, 2011).

Pour les sédiments de barrages ou voies d'eau, cas pour EDF, il est nécessaire d'avoir une vision intégratrice de l'ensemble des potentialités de valorisation sur le territoire français. Comme les ouvrages hydroélectriques et d'eau sont répartis, les gisements le sont aussi et parfois éloignés des activités humaines et industrielles. Il convient si possible de pouvoir valoriser ces sédiments au plus près de leur lieu de prélèvement. Il s'agit de limiter les coûts de transport et l'impact environnemental. Cette condition de proximité permet de répondre aux enjeux du développement durable. Selon les retenues, il faut tenir compte de la typicité des sédiments (minéralogie en relation avec le bassin versant). Intégrer condition de proximité, typicité et connaissance des sédiments, gestion durable et acceptabilité socio-économique constitue tout l'enjeu de la réponse à apporter à la valorisation des sédiments. Un élément-clé de la réponse est l'adéquation sédiments/filières. Pour ce faire, une démarche est proposée afin de définir une caractérisation pertinente, simple à mettre en place, économique et qui renseigne sur la compatibilité du sédiment avec les filières de valorisation. 


\section{XII ${ }^{\text {èmes }}$ Journées Nationales Génie Côtier - Génie Civil \\ Cherbourg, 12-14 juin 2012}

\subsection{Principe de la démarche}

Une démarche d'identification innovante des paramètres essentiels d'entrée dans les filières de valorisation est donc entreprise. En règle générale, valoriser un déchet nécessite de valider une succession d'étapes, afin de satisfaire l'ensemble des prescriptions techniques et environnementales du produit final envisagé.

La difficulté dans cette approche est de retenir la ou les données déterminantes ou discriminatoires. Le criblage sera positif ou négatif. On recense tant les données essentielles à toute filière de valorisation que celles qui constituent une impossibilité, données rédhibitoires pour la pré-orientation. L’objectif de cette démarche est donc de hiérarchiser les paramètres, du moins important ou pertinent, au paramètre clé. Pour ce faire, une liste complète des paramètres d'étude de la filière menant du sédiment $\mathrm{x}$ au produit final y est dressée, puis par analyse à rebours ou inverse (fig. 1), un classement des critères mènera à l'identification de paramètres-clés ou déclenchants pour cette voie de valorisation. Ainsi, seront dénombrés les critères essentiels à retenir. Cela impose bien évidemment de connaitre les filières et leurs prescriptions techniques dans leur ensemble.

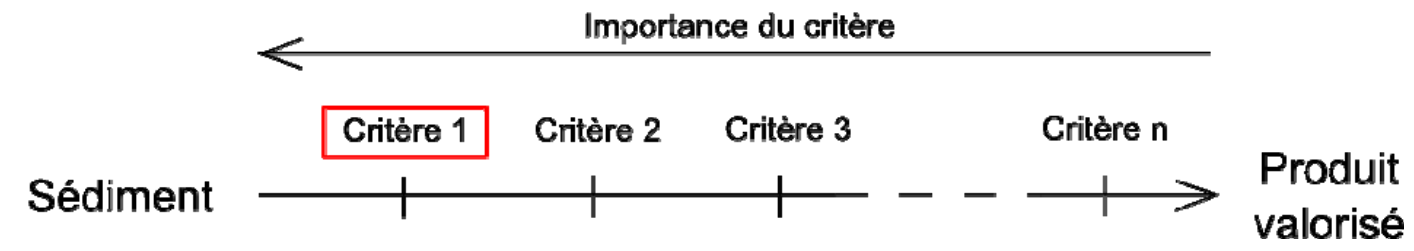

Figure 1. Principe de l'analyse inverse mise en place.

Ce travail de hiérarchisation des paramètres est réalisé prioritairement pour les filières prometteuses dans lesquelles les sédiments peuvent être valorisés dans des proportions importantes et à faible coût. Ainsi, il sera possible d'aboutir à une caractérisation minimale, rapide et à faible coût, et apporter une réponse quant à la valorisation possible des sédiments dans telle ou telle filière (phase de pré-orientation). Cette phase de préorientation, permet de classer les sédiments devant être gérés à terre, en constituant des familles organisées par typologies communes, identifiées grâce à la caractérisation minimale. C'est aussi la base pour la création d'un outil de décision sur le devenir des sédiments dragués gérés à terre.

\section{Organisation de la démarche : exemple d'application à un matériau routier}

Pour une filière de valorisation de type matériaux routiers, l'analyse des prescriptions techniques requises permet de déduire les caractérisations à entreprendre. Cette filière est intéressante de par les volumes de matériaux qu'elle consomme et du fait d'un manque de granulats éventuel dans les années à venir. De plus, le domaine routier 
exploite des matériaux de nature très variée qui demandent des caractéristiques de résistance mécanique peu élevées en fonction de la position du matériau dans la structure routière. De très nombreux travaux (COLIN, 2003; DUBOIS, 2006; SILITONGA, 2010 ; LIMEIRA, 2011), parfois en association avec des professionnels du secteur, ont étudié ce domaine. Dans certains cas, les travaux ont menés à la réalisation des tronçons routiers. Les techniques d'amélioration des sols fins (liants, correction granulométrique) ont fortement contribué au développement de la stabilisation/solidification des sédiments. L'exemple d'application retenu concerne une sous couche routière, appelée couche de forme.

\subsection{Prescriptions techniques pour une utilisation en couche de forme}

La couche de forme est un élément de transition permettant d'adapter les caractéristiques des matériaux de remblai ou de sols en place aux fonctions essentielles d'une plate-forme support de chaussée ou de voie. Elle doit donc répondre à des objectifs de court terme pour la réalisation de la chaussée ou de la voie (nivellement, portance, protection du sol support et traficabilité) et de long terme vis-à-vis de l'utilisation de l'ouvrage (homogénéisation, garantie d'une portance minimale, contribution au drainage de la plate-forme). Les prescriptions techniques relatives pour une utilisation en couche de forme sont consignées dans plusieurs guides: GTR (SETRA-LCPC, 1992), GTS (SETRA-LCPC, 2000) et récemment dans le guide méthodologique d'évaluation environnementale pour acceptabilité de matériaux alternatifs en technique routière (SETRA, 2011). Les normes de la profession viennent compléter les indications des guides. La démarche conventionnelle à suivre est :

- d'identifier le matériau [NF P 11-300 (AFNOR, 1992)] à partir des paramètres (granularité, argilosité, état hydrique) et de le classer. De par leur finesse, les sédiments sont classés comme équivalent à des sols fins ou comme sols organiques selon leur teneur en matière organique. Il est recommandé, pour ce type de matériau, fin, souvent plastique et parfois organique, de procéder à un traitement à base de liants.

- de vérifier l'aptitude au traitement [NF P 94-100 (AFNOR, 1999)]. Ces essais comprennent des mesures de gonflement volumique (Gv) et le cas échéant, de résistance mécanique avec un essai de traction indirecte (Rit).

- d'étudier les performances mécaniques du sédiment traité. Pour le cas d'une couche de forme, il faut se reporter à la norme NF P 94-102-2 (AFNOR, 2001), qui précise la méthodologie des études de formulations à suivre en laboratoire. Le comportement mécanique est à évaluer vis-à-vis de (i) l'âge autorisant la circulation sur la couche traitée, (ii) la résistance à l'immersion au jeune âge, (iii) la résistance au gel, (iv) et les performances escomptables à long terme. Toujours selon la norme NF P 94-102-2 (AFNOR, 2001), la résistance à l'immersion au jeune âge et la résistance au gel sont 


\section{XII ${ }^{\text {èmes }}$ Journées Nationales Génie Côtier - Génie Civil \\ Cherbourg, 12-14 juin 2012}

des actions optionnelles en fonction de l'environnement hydrique et du climat du site du chantier.

- d'évaluer l'acceptabilité environnementale en s'appuyant sur le guide SETRA (2011) qui propose une méthodologie avec notamment des essais de lixiviation et des seuils de référence.

Ainsi, une application en couche de forme nécessite la connaissance de paramètres d'identification (état, nature), de résistance mécanique, de durabilité et environnementaux.

4.2 Caractérisation : interaction entre paramètres et recherche du ou des paramètres-clés Même si les guides méthodologiques permettent de lister les essais nécessaires à mener pour déterminer un ensemble de paramètres et ce, dans le cas d'une application en couche de forme, il n'en demeure pas moins que des critères relatifs à des pré-requis sont à considérer. La teneur en eau en est un. En effet, une teneur en eau excessive se traduit par une portance médiocre, des caractéristiques de résistance faibles et une sensibilité au gel importante. C'est-à-dire que l'eau dans les sédiments à traiter peut constituer un frein à ce type de valorisation. Les sédiments à la sortie d'une drague ont une teneur en eau trop importante pour être utilisés directement. Ce point clairement identifié comme essentiel, est un verrou technique à fort impact économique sur la valorisation future (LEVACHER \& DHERVILLY, 2010). Il est donc important d'établir un critère relatif à la teneur en eau pour cette application de mise en couche de forme. Il est considéré que les sédiments auront subi un pré-traitement de type lagunage en bassin de décantation, une déshydratation mécanique à l'aide d'une unité spécifique ou encore un essorage à l'aide de tubes en géotextile selon la présence ou non d'espace disponible. La teneur en eau sera donc réduite, pour améliorer sa tenue physique, jusqu'à des conditions optimales de mise en œuvre.

Dans une démarche qui a pour but d'identifier les paramètres minimaux en vue d'une pré-orientation vers une filière de valorisation, les essais et analyses qui semblent essentiels de retenir sont détaillés dans ce qui suit. Il est possible de les regrouper en trois familles i.e. les paramètres d'identification, les paramètres de comportement mécanique et l'impact environnemental.

\subsubsection{Les paramètres d'identification}

Ils concernent :

- la granularité qui est un élément fondamental du classement GTR (SETRA-LCPC, 1992). Une analyse granulométrique est systématiquement réalisée dans les études de valorisation des sédiments fins dans le domaine routier.

- les limites d'Atterberg et l'argilosité. Pour évaluer l'argilosité, si l'indice de plasticité (Ip) est supérieur à 12, il devient plus adapté que la valeur au bleu, GTR (SETRALCPC, 1992). En effet, lorsqu'une plasticité est importante, l'indice de plasticité Ip 
est plus sensible que la valeur au bleu VBS. C’est le cas de la grande majorité des sédiments fins qui sont plastiques. De plus l'indice de plasticité est un paramètre d'identification mais aussi de comportement. En effet, l’Ip définit en réalité l'intervalle de teneur en eau dans lequel le sol reste souple et déformable tout en conservant une certaine résistance au cisaillement. La connaissance de cet intervalle est d'une manière générale très utile dans la conception des ouvrages en terre.

- la teneur en matière organique qui, en plus d'être un critère de classement dans le GTR (SETRA-LCPC, 1992), est également un élément qui affecte de façon importante les traitements à base de liants hydrauliques en faisant diminuer les résistances mécaniques (LEVACHER et al., 2006) et qui les rend donc inefficaces à un certain taux.

Ces trois types de paramètres sont en accord avec les conclusions de DUAN (2008), qui indiquent qu'une caractérisation minimale, pour toute valorisation, comprend la répartition dimensionnelle, la nature (\% des classes), les teneurs en matière organique et les limites d'Atterberg.

\subsubsection{Les paramètres de comportement mécanique}

On peut retenir comme essais d'importance ou indicateurs de référence :

- la résistance à la compression (Rc), qui dans l'exemple retenu pour une utilisation en couche de forme, doit atteindre 1 MPa, (GTS, 2000 ; AFNOR-NF P 94-102-2, 2001). C’est le critère de traficabilité autorisant la circulation du chantier ; la résistance à la compression simple (Rc) est aussi un indicateur de l'intégrité physique d'un matériau solidifié. Dans la cadre du projet SEDIMARD83, SANNIER et al (2009) utilisent la résistance à la compression à 28 jours comme indicateur de l'efficacité de multiples traitements appliqués sur différents sédiments portuaires méditerranéens. La résistance à la compression permet d'une part, de déterminer un dosage optimal en liant(s) à utiliser pour obtenir le critère de portance (ou de résistance) et d'autre part de déterminer le temps de cure nécessaire permettant de garantir certaines performances comme la traficabilité.

- le couple résistance à la traction indirecte/module de déformation (Rt/E) est à considérer. Il permet de connaitre les performances mécaniques à long terme. Ce couple est presque systématiquement mesuré dans les travaux de valorisation de sédiments fins pour une couche de forme (LEVACHER et al., 2006 ; SIHAM et al., 2008 ; ZENTAR et al., 2008; DUBOIS et al., 2009; OBANA et al., 2010 ; BOUTOUIL et al., 2011 ; TRIBOUT \& HUSSON, 2011; WANG et al., 2011 ; MIRAOUI et al., 2012). Si ces essais apparaissent nécessaires pour une validation de sédiments traités pour une application en couche de forme, il faut valider en amont un seuil de résistance Rc minimal. 


\section{XII ${ }^{\text {èmes }}$ Journées Nationales Génie Côtier - Génie Civil \\ Cherbourg, 12-14 juin 2012}

\subsubsection{L'impact environnemental}

Pour l'application en couche de forme, il faut aussi vérifier l'absence d'impacts environnementaux. Le guide d'adaptabilité des matériaux alternatifs en technique routière (SETRA, 2011), recommande comme premier niveau d'étude de faire un test de lixiviation et propose des seuils à respecter.

\subsubsection{Proposition de hiérarchisation des paramètres}

La démarche est, à partir d'une liste de paramètres nécessaires à une filière de valorisation, de les hiérarchiser de manière à isoler les paramètres-clés qui pourront servir à pré-orienter les sédiments vers telle ou telle filière.

On aboutit donc à une première proposition de hiérarchisation des paramètres identifiés comme essentiels (fig. 2). Cette proposition doit maintenant être discutée avec les professionnels et universitaires.

Les paramètres listés sont seulement identifiés pour réaliser une pré-orientation. Audelà, il faudra une caractérisation complémentaire afin de satisfaire tous les critères de la filière proposée.

\begin{tabular}{c|cccc} 
& \multicolumn{4}{c}{ Importance du critère } \\
\cline { 2 - 5 } $\begin{array}{c}\text { Sédiment } \\
\text { brut }\end{array}$ & Ip & \%MO & Granularité & $\% w$ \\
\hline $\begin{array}{c}\text { Sédiment } \\
\text { traité }\end{array}$ & & Test de lixiviation & Rit/E & Rc
\end{tabular}

Figure 2. Proposition de classement des paramètres essentiels.

\section{Conclusion et perspectives}

Les résultats apportés par cette approche participeront à l'amélioration des connaissances de la gestion à terre des sédiments, étape indispensable à l'évolution du cadre réglementaire, à l'émergence et à la pérennisation de filières terrestres de "valorisation matière" appliquées aux sédiments.

Pour que les voies de valorisation deviennent robustes et donc pérennes, il faudra aussi considérer les coûts inhérents à la filière de valorisation y compris les coûts relatifs de la mise à disposition des sédiments avant traitement (dragage, déshydratation et transport), les volumes en relation avec les capacités d'absorption des filières dans le temps, l'aspect normatif et réglementaire. En effet, il existe un panel de voies potentielles ayant chacune des contraintes particulières, cependant, les textes prescriptifs n'existent pas pour le cas spécifique des sédiments. Il faut, en général, se référer aux textes prescriptifs applicables à des filières similaires. Des propositions d'aménagement de ces textes ou de mise au point de guides est prévue. 


\section{Références bibliographiques}

AFNOR (1992). NF P 11-300 - Exécution des terrassements - Classification des matériaux utilisables dans la construction des remblais et des couches de forme d'infrastructures routières. $21 \mathrm{p}$.

AFNOR (1999). NF P 94-100 - Sols : Reconnaissance et essais - Matériaux traités à la chaux et/ou aux liants hydrauliques - Essai d'évaluation de l'aptitude d'un sol au traitement. $12 \mathrm{p}$.

AFNOR (2001). NF P 94-102-2 - Sols : Reconnaissance et essais - Sol traité au liant hydraulique, éventuellement associé à la chaux, pour utilisation en couche de forme Partie 2 : Méthodologie des études de formulation en laboratoire. 13 p.

BOUTOUIL M., BEN ABDELGHANI F., SAUSSAYE L., MAHERZI W. (2011). Étude du comportement des sédiments de dragage marins à l'essai d'aptitude au traitement. $2^{\text {ème }}$ Conférence Méditerranéenne Côtière et Maritime, 22-24 novembre, Tanger, Maroc, pp 141-144. doi:10.5150/cmcm.2011.030

COLIN D. (2003). Valorisation de sédiments fins de dragage en technique routière. Thèse de doctorat, Université de Caen. 181 p.

DUAN Z. (2008). Caractérisation, stabilisation et solidification de sédiments fins marins. Thèse de doctorat. Université de Caen. 145 p.

DUBOIS V. (2006). Etude du comportement physico-mécanique et caractérisation environnementale des sédiments marins : valorisation en technique routière. Thèse de doctorat, ENSM de Douai, 291 p.

DUBOIS V., ABRIAK N.E., ZENTAR R., BALLIVY G. (2009). The use of marine sediments as a pavement base material. Waste Management, n²9, pp 774-782.

EDF (2011). Le guide biodiversité hydraulique. Document interne. 88 p.

EDF/IME (2010). Rapport régional sur la gestion des sédiments dans les barrages en méditerranée. Atelier régional, 13 et 14 décembre 2010, Marseille, France. 245p.

EDF (1997). Gestion des dépôts des ouvrages hydrauliques. Document interne.

JOCE (2000). Directive $n^{\circ}$ 2000/60/CE du 23/10/00 établissant un cadre pour une politique communautaire dans le domaine de l'eau. Journal Officiel des Communautés Européens $\mathrm{n}^{\circ} \mathrm{L} 327$ du 22 décembre 2000.

JOFR (1994). Arrêté du 22/09/94 relative aux exploitations de carrières et aux installations de premier traitement des matériaux de carrières. Journal Officiel de la République Française n 246 du 22 octobre 1994.

JORF (2006). Loi n 2006-1772 du 30/12/06 sur l'eau et les milieux aquatiques. Journal Officiel de la République Française n 303 du 31 décembre 2006 et Rectificatif au JO $\mathrm{n}^{\circ} 17 \mathrm{du} 20$ janvier 2007.

JORF (2010). Décret $n^{\circ}$ 2010-367 du 13 avril 2010 modifiant la nomenclature des installations classées et ouvrant certaines rubriques au régime de l'enregistrement. Journal Officiel de la République Française n87 du 14 avril 2010. 


\section{XII ${ }^{\text {èmes }}$ Journées Nationales Génie Côtier - Génie Civil \\ Cherbourg, 12-14 juin 2012}

BO (2011). Circulaire du 24/12/10 relative aux modalités d'application des décrets $n^{\circ}$ 2009-1341, 2010-369 et 2010-875 modifiant la nomenclature des installations classées exerçant une activité de traitement de déchets. BO du MEDDTL nº 1 du 25/01/2011.

LEVACHER D., DHERVILLY P. (2010). Déshydratation mécanisée in situ de sédiments fraîchement dragués ou mis en dépôts : le projet SEDIGATE I. XIèmes Journées Nationales Génie Côtier - Génie Civil, 22-25 juin 2010, Les Sables d’Olonne, pp 859-868. doi:10.5150/jngcgc.2010.093-L

LEVACHER D., COLIN D., PERRONI A.C., DUAN Z., SUN, L. (2006). Recyclage et valorisation de sédiments fins de dragage à usage de matériaux routiers. IX ${ }^{\text {èmes }}$ Journées Nationales Génie Civil - Génie Côtier, 12-14 septembre 2006, Brest, pp 603-612. doi:10.5150/jngcgc.2006.058-L

LEVACHER D., BATAGIN B., LIANG Y. (2012). Reuse of waste in sediments and fillers stabilization: attempts, performances and problems. International Conference on Sediment Management 2012, Alibaug, India, 20-22 March 2012.

LIMEIRA J., (2011). The beneficial use of dredged marine sand as an alternative material for concrete. Ph. D Thesis, Polytechnic University of Catalonia, $191 \mathrm{p}$.

MIRAOUI M., ZENTAR R., ABRIAK N.-E. (2012). Road material basis in dredged sediment and basic oxygen furnace steel slag. Construction and Building Materials, n³0, pp 309-319. doi:10.1016/j.conbuildmat.2011.11.032

OBANA M., LEVACHER D., DHERVILLY P. (2010). Durability properties of marine sediment stabilized by pozzolan and alkali activated binders. XI ${ }^{\text {èmes }}$ Journées Nationales Génie Côtier - Génie Civil, Les Sables d'Olonne, 22-25 juin 2010 pp 887-894. doi:10.5150/jngcgc.2010.096-O

PAIPAI E., (1995). Environmental enhancement using dredged material. Terra Aqua n०59, pp 5-21.

PAIPAI E., (2003). Beneficial uses of dredged material: Yesterday, today and tomorrow. Terra Aqua n92, pp 3-12

SANNIER L., LEVACHER D., JOURDAN M. (2009). Approche économique et validation de méthodes de traitements aux liants hydrauliques de sédiments marins contaminés. Revue Paralia, n²2, s2.1-s2.15. doi:10.5150/revue-paralia.2009.s02

SETRA-LCPC, (1992). Guide Technique - Réalisation des remblais et des couches de forme, Fascicule 1, principes généraux. $100 \mathrm{p}$.

SETRA, LCPC (2000). Guide Technique - Traitement des sols à la chaux et/ou aux liants hydrauliques : application à la réalisation des remblais et des couches de forme. $240 \mathrm{p}$.

SETRA (2011). Acceptabilité de matériaux alternatifs en technique routière, Evaluation environnementale guide méthodologique. $32 \mathrm{p}$.

SIHAM K., FABRICE B., ABRIAK N.E., PATRICK D. (2008). Marine dredged sediments as new materials resource for road construction. Waste Management $\mathrm{n}^{\circ} 28$, pp 919-928. 
Thème 8 - Gestion et valorisation des sédiments marins

THERY F., ANGER B. (2011) Sédiments fins de barrage : cadre réglementaire, identification des voies de valorisation et caractérisation minéralogique. EDF R\&D. Document interne, $51 \mathrm{p}$.

TRIBOUT C., HUSSON B. (2011). Use of treated sediments in road building technique, European Journal of Environmental and Civil Engineering, volume 15, $n^{0} .2$ pp, 197-213. doi:10.3166/ejece.15.197-213

WANG D., ABRIAK N.E., ZENTAR R. (2011). Durability analysis of fly ash/cementsolidified dredged materials. ${ }^{2 \mathrm{ème}}$ Conférence Méditerranéenne Côtière et Maritime, 2224 novembre, Tanger, Maroc, pp 245-250. doi:10.5150/cmcm.2011.053

ZENTAR R., DUBOIS V., ABRIAK N.E. (2008). Mechanical behaviour and environmental impacts of a test road built with marine dredged sediments. Resources, Conservation and Recycling, n52, pp 947-954. doi:10.1016/j.resconrec.2008.02.002 